\title{
Modified Eye Evisceration in a Tropical Screech Owl (Megascops choliba)
}

\author{
João Antonio Tadeu Pigatto, Eduarda Valim Borges de Vargas, Marcela Torikachvili, \\ Alessandra Fernandez da Silva, Renata Lima Baptista, Maiara Poersch Seibel \& Daniela Nicknich
}

\begin{abstract}
Background: An adult owl was presented with an injury to the right eye that rendered it blind in that eye. The left eye was normal. Removal of the right eye was recommended and a modified eye evisceration was performed. No complications were observed during or after surgery. The objective of this paper is to describe the modified eye evisceration technique that was successfully used in a tropical screech owl (Megascops choliba).

Case: An adult owl was presented with an injury to the right eye that rendered it blind in that eye. Two previous surgical treatments have been carried out but have not been successful. Using a portable slit-lamp (Kowa SL-15 ${ }^{\circledR}$ ), both eyes were examined. The left eye was normal. Upon ophthalmic examination of the right eye, the owl demonstrated blepharospasm and large central corneal ulcer. Removal of the right eye was recommended. The bird received midazolam hydrochloride [Dormire ${ }^{\circledR}-1 \mathrm{mg} / \mathrm{kg}, \mathrm{IM}$ ] and ketamine hydrochloride [Ketamina ${ }^{\circledR}-5 \mathrm{mg} / \mathrm{kg} \mathrm{IM}$ ] as pre-anesthetic medications. Subsequently, the bird was anesthetized with isoflurane (Isoforine ${ }^{\circledR}$ ) by facemask for induction, and then maintained with isoflurane vaporized in 100\% oxygen through an endotracheal tube. With the aid of a surgical microscope and microsurgery materials, a modified eye evisceration was performed. Post-operatively, the owl received meloxicam [Maxicam ${ }^{\circledR}-0.5 \mathrm{mg} / \mathrm{kg}$, IM] and tramadol hydrochloride [Cronidor ${ }^{\circledR}-15 \mathrm{mg} / \mathrm{kg}$, orally for 4 days]. The day after surgery, the owl was comfortable and its usual appetite was regained. The patient remained hospitalized for 3 weeks and was evaluated daily. The skin sutures were removed 10 days after the surgical procedure and the surgical wound had healed normally. The patient was reintroduced into the wild after 2 months. During the 6 months post-release, the bird was evaluated once a month, and no complications were observed.

Discussion: Severe eye trauma and complicated corneal ulcers are common causes of eyeball removal in birds. In birds, there is a high risk of complications during enucleation. The fragility of the orbital bones makes them susceptible to trauma during the surgery. Evisceration involves the removal of the inner contents of the eye while leaving the cornea and the sclera intact. In the current case, evisceration was chosen because the eye was blind, and maintaining a blind eye would be a source of pain and infection. In the modified evisceration technique, the risk of complications is minimal compared to enucleation, mainly because surgical manipulation is minimal. In our case, the total surgery time was 20 min. Another complication reported after enucleation in birds is the possibility of disfiguring the bird because the removal of the globe disturbs the natural head balance. To avoid these complications, the use of an intraocular prosthesis after evisceration in birds has been performed. However, owls have a tubular-shaped globe with scleral ossicles. These factors could hinder or even prevent the accommodation of a cylindrical silicone prosthesis. In the present case, an intraocular prosthesis implant was never considered due to the unavailability of the prosthesis and to avoid the risk of postoperative complications that have been reported from the literature in dogs. In this case, the owl recovered well from anesthesia without complications, and no postoperative hemorrhage was observed. No signs of pain were observed during the postoperative period and the owl had already shown an appetite and fed on the first postoperative day. The previously published reports using the modified evisceration technique also demonstrated an absence of pain signs during the postoperative period.
\end{abstract}

Keywords: wild, avian, surgery, ophthalmology, enucleation. 


\section{INTRODUCTION}

Among the main indications for removing the eye in birds are mainly eye trauma, endophthalmitis, glaucoma, and intraocular neoplasia [2]. Normally, enucleation is a procedure commonly used in birds [9]. However, complications associated with extraocular dissection, hemorrhage, and lesions in the optic chiasm and orbital bones have been observed $[1,9]$. Additionally, enucleation causes an asymmetric weight distribution in the bird's head [4]. Despite the large number of complications that occur during enucleation in birds, this technique is still the most used [9]. Modified eye evisceration has been employed as an alternative to enucleation in birds $[1,2,5,9]$. In this technique, the cornea and all intraocular contents are removed and an intrascleral prosthesis is not implanted [1]. Compared to enucleation, numerous advantages have been attributed to modified eye evisceration, including decreased anesthetic time, ease of procedure, decreased risk of excessive traction of the optic nerve, decreased intraoperative orbital trauma, and preservation of the natural symmetry of the head [2,9]. Despite the promising results of modified eye evisceration in birds, the documented cases are relatively scarce in the literature $[1,2,5,9]$. No previous documentation has been found in the literature regarding eyeball removal in tropical screech owls. In this sense, the objectives of this paper are to describe the modified eye evisceration technique used successfully in a tropical screech owl and to increase the visibility of safer techniques for eye surgery in birds.

\section{CASE}

An adult tropical screech owl (Megascops choliba) with a bodyweight of $165 \mathrm{~g}$ and a was referred to the Ophthalmology Veterinary Section of the Federal University of Rio Grande do Sul (UFRGS), Porto Alegre, Brazil for evaluation of a corneal lesion. The corneal ulcer occurred one month prior to presentation (Figure 1). Two previous surgical treatments were attempted, but it was unsuccessful. The physical examination was unremarkable. Upon ophthalmic examination of the right eye, the owl demonstrated blepharospasm and tearing. Using a portable slit-lamp (Kowa SL-15 $\left.{ }^{\circledR}\right)^{1}$, both eyes were examined. An ophthalmic examination of the right eye revealed blepharospasm, epiphora, and a deep central corneal ulcer. It was not possible to evaluate the intraocular structures due to the severe corneal edema. The right eye had an intraocular pressure (IOP) of $3 \mathrm{mmHg}$ as measured with rebound tonometry $\left(\mathrm{TonoVe}^{\circledR}\right)^{2}$. Ophthalmic examination of the left eye appeared normal and the IOP was $16 \mathrm{mmHg}$. Because the ownl was blind and painful in the right eye removal of the eyeball was recommended. A blood collection was performed; the blood count and biochemical tests were within the normal range for the species. After a solid fast of approximately $6 \mathrm{~h}$, the bird received midazolam hydrochloride ${ }^{3}(1 \mathrm{mg} / \mathrm{kg}$, IM) and ketamine hydrochloride ${ }^{4}(5 \mathrm{mg} / \mathrm{kg}$ IM) as pre-anesthetic medications. Subsequently, the bird was anesthetized with isoflurane ${ }^{3}$ by facemask for induction, and then maintained with isoflurane vaporized in $100 \%$ oxygen through an endotracheal tube. The patient was then placed in lateral recumbency on a heating pad, and the surgical area was aseptically prepared for surgery utilizing a 1:50 ratio of povidone to iodine. The surgical procedure was performed using a surgical microscope. A lateral canthotomy was performed and a Barraquer eyelid speculum was placed under the eyelids. The cornea was incised with a microsurgery knife and removed along the limbus using corneal scissors. A cyclodialysis spatula was used to dissect the sclera from the uvea.

The uvea was grasped with forceps, and the remaining intraocular structures (uvea, lens, and vitreous body) were removed from the eye. An absorbable hemostatic gelatin sponge ${ }^{5}$ was placed inside the scleral shell. The nictitating membrane and the upper and lower eyelid edges were excised with iris scissors. The subcutaneous tissues were closed with a simple continuous suture pattern using polyglactin $7-0^{6}$. The skin was closed with 5-0 polypropylene ${ }^{6}$ in a simple interrupted pattern. The owl recovered without complications from

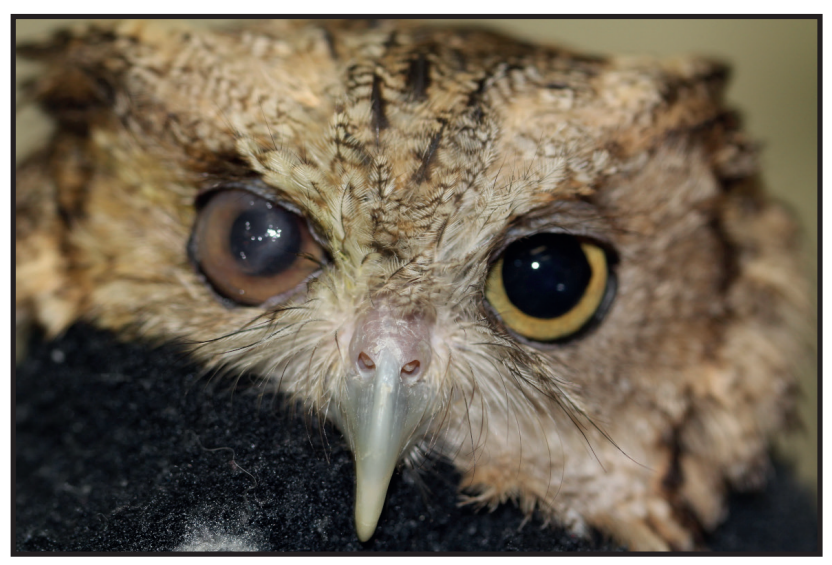

Figure 1. Appearance of the tropical screech that was presented with corneal ulcer the right eye. 
anesthesia. Post-operatively, the owl received meloxi$\mathrm{cam}^{7}(0.5 \mathrm{mg} / \mathrm{kg}, \mathrm{IM})$ and tramadol hydrochloride ${ }^{4}(15$ $\mathrm{mg} / \mathrm{kg}$, orally) for 4 days. The day after surgery, the owl was comfortable and its usual appetite was regained; it was defecating normally. The patient remained hospitalized for 3 weeks and was evaluated daily. The skin sutures were removed 10 days after the surgical procedure and the surgical wound had healed normally. The patient was reintroduced into the wild after 2 months. During the 6 months post-release, the bird was evaluated once a month, and no complications were observed. The aesthetic result was excellent (Figure 2).

\section{DISCUSSION}

Severe eye trauma and complicated corneal ulcers are common causes of eyeball removal in birds [9]. Enucleation is still frequently performed in birds even though numerous iatrogenic complications from this surgical procedure have been reported [9]. In birds, there is a high risk of complications during enucleation. The fragility of the orbital bones makes them susceptible to trauma during enucleation; furthermore, the optic nerve is short and can result in blindness of the contralateral eye due to excessive traction of the eyeball [1]. The interorbital septum is very thin in owls, which provides a risk of additional damage to the contralateral eye during enucleation [9].

Evisceration involves the removal of the inner contents of the eye while leaving the cornea and the sclera intact $[6,8]$. In this procedure, an implant is often placed inside the sclera in order to maintain the shape of the eyeball $[6,8]$. In the current case, evisceration was chosen because the eye was blind, and maintaining a blind eye would be a source of pain and infection. In addition, a maintained blind eye could cause future complications for the owl. Evisceration is contraindicated if an intraocular tumor is suspected $[6,8]$.

Although the literature on neoplasms in birds is relatively scarce, a variety of types of eye tumors can affect the eyes of birds [3]. In cases of suspected intraocular neoplasia, evisceration is contraindicated to prevent the spread of unexpected intraocular neoplasia. In the modified evisceration technique, the risk of complications is minimal compared to enucleation, mainly because surgical manipulation is minimal [2]. In our case, the total surgery time was $20 \mathrm{~min}$. Dees et al. [2] performed the modified evisceration on an eagle in $15 \mathrm{~min}$. It is likely that with a larger number of cases, this time may decrease even more.

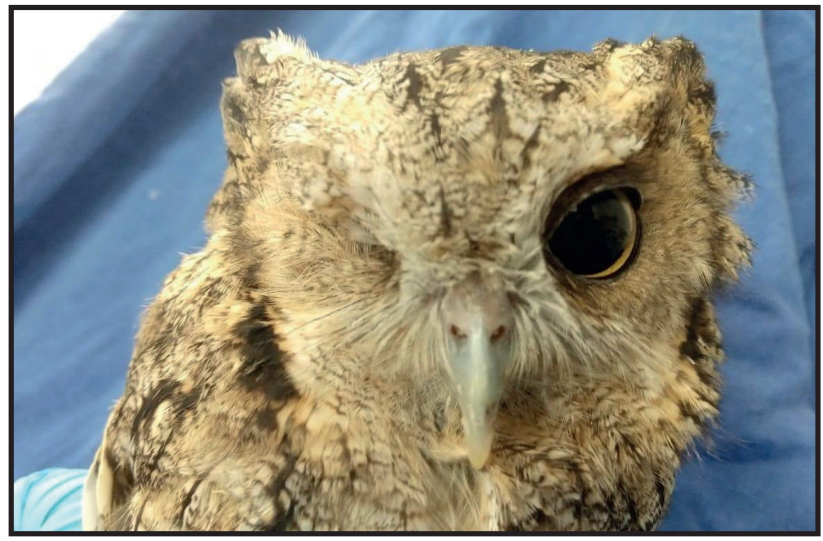

Figure 2. Appearance of the tropical screech owl 6 months after the modified eye evisceration of the right eye.

Another complication reported after enucleation in birds is the possibility of disfiguring the bird because the removal of the globe disturbs the natural head balance [10]. To avoid these complications, the use of an intraocular prosthesis after evisceration in birds has been performed [4,10]. In dogs, prostheses with a diameter between 16 and $22 \mathrm{~mm}$ are usually implanted $[6,8]$. However, owls have a tubular-shaped globe with scleral ossicles. These factors could hinder or even prevent the accommodation of a cylindrical silicone prosthesis. Graham et al. [4] used a silicone prosthesis after an owl was eviscerated. Modifications were made to the evisceration technique used in dogs to accommodate the prosthesis inside the tubular eyeball in birds. Graham et al. [4] used 2 silicone prostheses at 12 and $22 \mathrm{~mm}$ in diameter after an owl was eviscerated. The injection of silicone oil after evisceration has been reported as an alternative to placing a rigid prosthesis in birds [10]. Fluid silicone oil made it possible to fill the owl's entire tubular eyeball. This was conducted by the authors as an advantage over a spherical prosthesis [10].

In the present case, an intraocular prosthesis implant was never considered due to the unavailability of the prosthesis and to avoid the risk of postoperative complications that have been reported from the literature in dogs [10]. Intraocular silicone prostheses have often been used after evisceration in dogs $[6,8]$. However, this technique is not exempt from postoperative complications. Complications have been reported after the implantation of an intraocular prosthesis in dogs, including decreased tear production, corneal ulcer, prosthesis rejection, suture dehiscence, and endophthalmitis [6,8]. In the 3 articles consulted from the literature on birds where intraocular prosthesis 
implantation was performed after evisceration, postsurgical corneal ulcers were reported as a complication in all cases $[4,10]$.

Mortality from general anesthesia is generally higher in birds than in other species. The main risk from inhalation anesthesia in birds is hypothermia, especially in smaller species [7]. Other anesthetic techniques have been developed in birds species [7]. In the current case, this procedure provided proper anesthesia without complications. To minimize the risk of heat loss, the bird was placed on a heating pad. In the current case, this procedure provided proper anesthesia without complications.

The modified evisceration technique chosen in the present case was the same used in a sulfur-crested cockatoo [1]. This same modified evisceration procedure has also been described in a golden eagle (Aquila chrysaetos) [2] and a blue-fronted amazon (Amazona aestiva) [5]. Murray et al. [9] described a series of cases where the modified evisceration technique was used in 19 birds of prey. In a retrospective study by Murray et al. [9], the only complication observed during the surgical procedure was a moderate hemorrhage, which was controlled by placing absorbable gelatin in the intraocular space. Dees et al. [2] and Gralla et al. [5] also reported the occurrence of a trans-operative hemorrhage. In our case, the only complication observed during the surgical procedure was a moderate hemorrhage, which was controlled by placing absorbable hemostatic gelatin sponges inside the scleral shell. In all cases described in the literature, the measure adopted to control bleeding during surgery was the same. The sponges were left in place in all cases. Cellulose sponges were not used in a single case of the evisceration of a sulfur-crested cockatoo [1]. Although this has not been done, complications related to hemorrhage were not reported during that surgical procedure [1].

In the present case, the owl recovered well from anesthesia without complications, and no postoperative hemorrhage was observed. Possible postoperative complications of eyeball removal have been reported, including suture dehiscence, infection, and hemorrhage [2]. In the present report, no postoperative complications were observed. No signs of pain were observed during the postoperative period and the owl had already shown an appetite and fed on the first postoperative day. The previously published reports using the modified evisceration technique also demonstrated an absence of pain signs during the postoperative period $[2,5,9]$.

In the modified evisceration technique, minimal manipulations of the eyeball and extraocular structures are done [2]. In the current case, the postoperative period was managed with a non-steroidal anti-inflammatory and an analgesic. Similar analgesic protocols have been used successfully in other birds submitted to the removal of the eyeball with excellent results $[5,9]$. In conclusion, the modified eye evisceration technique was efficient for allowing the rapid recovery and reintroduction of the animal to the wild.

\section{MANUFACTURERS}

${ }^{1}$ Kowa Company Ltd. Aichi, Japan.

${ }^{2}$ ICare Finland Oy. Helsinki, Finland.

${ }^{3}$ Cristália Produtos Químicos Farmacêuticos Ltda. São Paulo. SP, Brazil.

${ }^{4}$ Agener União. Embu-Guaçu. SP, Brazil.

${ }^{5}$ Laboratórios Pfizer Ltda. Guarulhos, SP, Brazil.

${ }^{6}$ Johnson do Brasil Indústria e Comércio de Produtos para Saúde Ltda. São Paulo, SP, Brazil.

${ }^{7}$ Ouro Fino Saúde Animal. Cravinhos, SP, Brazil.

Declaration of interest. The authors report no conflicts of interest. The authors alone are responsible for the content and writing of the paper.

\section{REFERENCES}

1 Christen C., Richter M., Fischer I., Eule C., Spiess B. \& Hatt J.M. 2006. Unilateral evisceration of an eye following cornea and lens perforation in a sulfur-crested cockatoo (Cacatua galerita). Schweizer Archiv für Tierheilkunde. 148(11): 615-619.

2 Dees D.D., Knollinger A.M. \& Maclaren N.E. 2011. Modified evisceration technique in a golden eagle (Aquila chrysaetos). Veterinary Ophthalmology. 14(5): 341-344.

3 Fernandez J.RR. \& Dubielzig R.R. 2014. Ocular and eyelid neoplasia in birds: 15 cases (1982-2011). Veterinary Ophthalmology. 18(s1): 113-118.

4 Graham J.E., Larocca R.D. \& Mclaughlin S.A. 1999. Implantation of an intraocular silicone prosthesis in a great horned owl (Bubo virginianus). Journal of Avian Medicine and Surgery. 13(2): 98-103.

5 Gralla S., Fischer D., Olerth D.V., Lierz M. \& Kramer M. 2015. Modified evisceration in a blue-fronted amazon (Amazona aestiva) as an ocular treatment option in parrots. Journal of Exotic Pet Medicine. 25(1): 7-12. 
J.A.T. Pigatto, E.V.B. Vargas, M. Torikachvili, et al. 2021. Modified Eye Evisceration in a Tropical Screech Owl (Megascops choliba). Acta Scientiae Veterinariae. 49(Suppl 1): 664.

6 Hamor R.E., Whitley R.D., Mclauglhin S.A., Lindley D.M. \& Albert R.A. 1994. Intraocular silicone prostheses in dogs: a review of the literature and 50 new cases. Journal of American Animal Hospital Association. 30(1): 66-69.

7 Kazemi-darabadi S., Akbari G. \& Shokrollahi S. 2019. Development and evaluation of a technique for spinal anaesthesia in broiler chickens. New Zealand Veterinary Journal. 67(5): 241-248.

8 Mclaughlin S.A., Gilger B.C., Hamilton H.L. \& Whitley R.D. 1995. Intraocular silicone prosthesis. Compendium on Continuing Education for the Practicing Veterinarian. 17(7): 945-950.

9 Murray M., Pizzirani S. \& Tseng F. 2013. A technique for evisceration as an alternative to enucleation in birds of prey: 19 cases. Journal of Avian Medicine and Surgery. 27(2): 120-127.

10 Sandmeyer L., Breaux C., Mcruer D. \& Grahn B. 2007. Case report: a new technique for intraocular prosthesis implantation in a great horned owl (Bubo virginianus). Journal of Exotic Pet Medicine. 16(2): 95-100. 